\title{
An epitope tag alters phosphoglycerate dehydrogenase structure and impairs ability to support cell proliferation
}

\author{
Katherine R Mattaini ${ }^{1,2}$, Edward J Brignole ${ }^{3,4}$, Mitali Kini ${ }^{1,2}$, Shawn M Davidson ${ }^{1,2}$, Brian P Fiske ${ }^{1,2}$, \\ Catherine L Drennan ${ }^{2,3,4}$ and Matthew G Vander Heiden ${ }^{1,2,5,6^{*}}$
}

\begin{abstract}
Background: The gene encoding the serine biosynthesis pathway enzyme PHGDH is located in a region of focal genomic copy number gain in human cancers. Cells with PHGDH amplification are dependent on enzyme expression for proliferation. However, dependence on increased PHGDH expression extends beyond production of serine alone, and further studies of PHGDH function are necessary to elucidate its role in cancer cells. These studies will require a physiologically relevant form of the enzyme for experiments using engineered cell lines and recombinant protein.

Results: The addition of an N-terminal epitope tag to PHGDH abolished the ability to support proliferation of PHGDH-amplified cells despite retention of some activity to convert 3-PG to PHP. Introducing an R236E mutation into PHGDH eliminates enzyme activity, and this catalytically inactive enzyme cannot support proliferation of PHGDH-dependent cells, arguing that canonical enzyme activity is required. Tagged and untagged PHGDH exhibit the same intracellular localization and ability to produce D-2-hydroxyglutarate (D-2HG), an error product of PHGDH, arguing that neither mislocalization nor loss of D-2HG production explains the inability of epitope-tagged PHGDH to support proliferation. To enable studies of PHGDH function, we report a method to purify recombinant PHGDH and found that untagged enzyme activity was greater than N-terminally tagged enzyme. Analysis of tagged and untagged PHGDH using size exclusion chromatography and electron microscopy found that an $\mathrm{N}$-terminal epitope tag alters enzyme structure.
\end{abstract}

Conclusions: Purification of untagged recombinant PHGDH eliminates the need to use an epitope tag for enzyme studies. Furthermore, while tagged PHGDH retains some ability to convert 3PG to PHP, the structural alterations caused by including an epitope tag disrupts the ability of PHGDH to sustain cancer cell proliferation.

Keywords: Phosphoglycerate dehydrogenase, Serine metabolism, Serine synthesis, Cancer Metabolism, Epitope tag

\section{Background}

Flux through the pathway that synthesizes serine from glucose is important for proliferation of some cancer cells. D-3-phosphoglycerate dehydrogenase (PHGDH) catalyzes the first reaction in serine biosynthesis. Using $\mathrm{NAD}^{+}$as a cofactor, PHGDH oxidizes the glycolytic intermediate 3phosphoglycerate (3-PG) to phosphohydroxypyruvate (PHP) (Figure 1A). Subsequent steps in serine biosynthesis involve

\footnotetext{
* Correspondence: mvh@mit.edu

${ }^{1}$ Koch Institute for Integrative Cancer Research, Massachusetts Institute of Technology, Cambridge, MA 02139, USA

${ }^{2}$ Department of Biology, Massachusetts Institute of Technology, Cambridge, MA 02139, USA

Full list of author information is available at the end of the article
}

phosphoserine aminotransferase 1 (PSAT1)-catalyzed transamination of PHP and glutamate to phosphoserine (P-Ser) and alpha-ketoglutarate $(\alpha \mathrm{KG})$, followed by phosphoserine phosphatase (PSPH)-mediated dephosphorylation of P-Ser to produce serine. Serine has multiple fates in cells, serving as an amino acid for protein synthesis and a precursor for lipid head groups. Serine can also be further metabolized to glycine by serine hydroxymethyltransferase, which donates a one-carbon unit to the folate pool for nucleotide synthesis and methyltransferase reactions. In addition to its role as an amino acid, glycine also contributes to nucleotide 
A

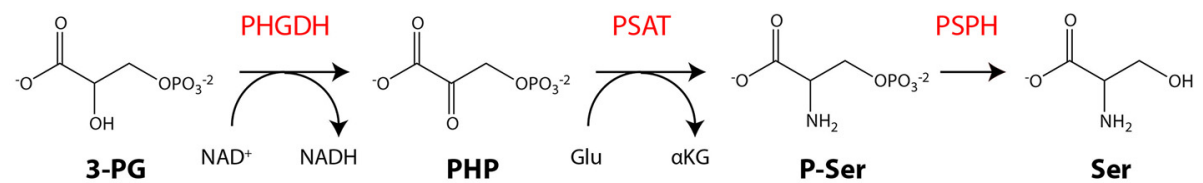
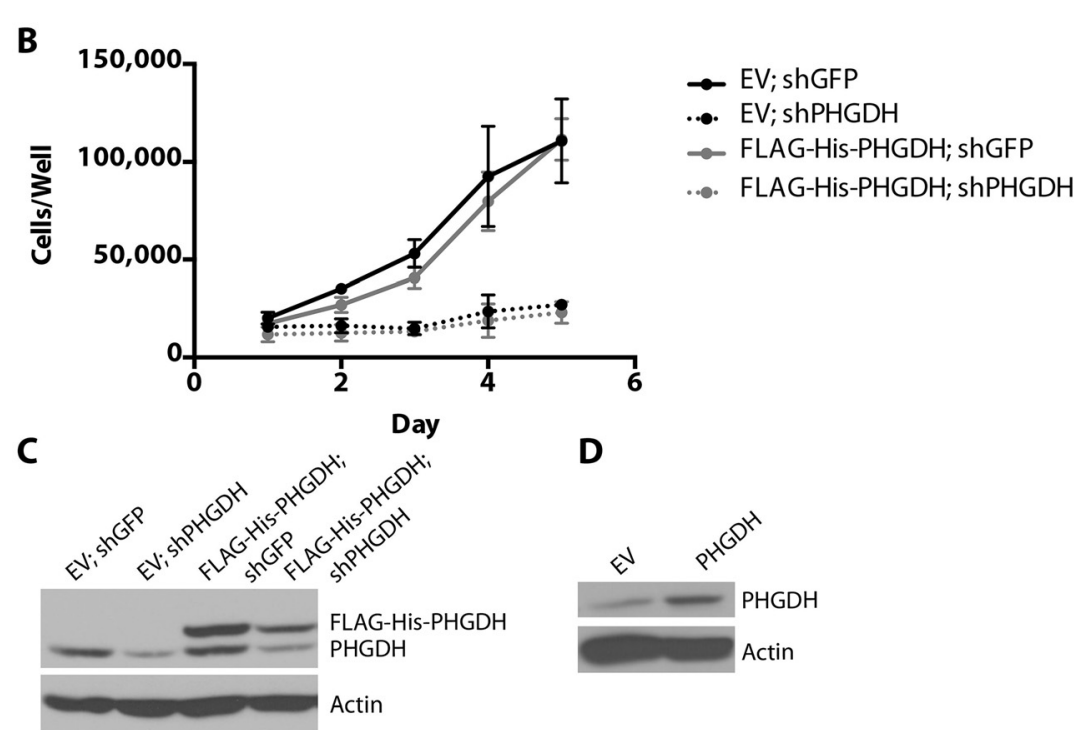

D

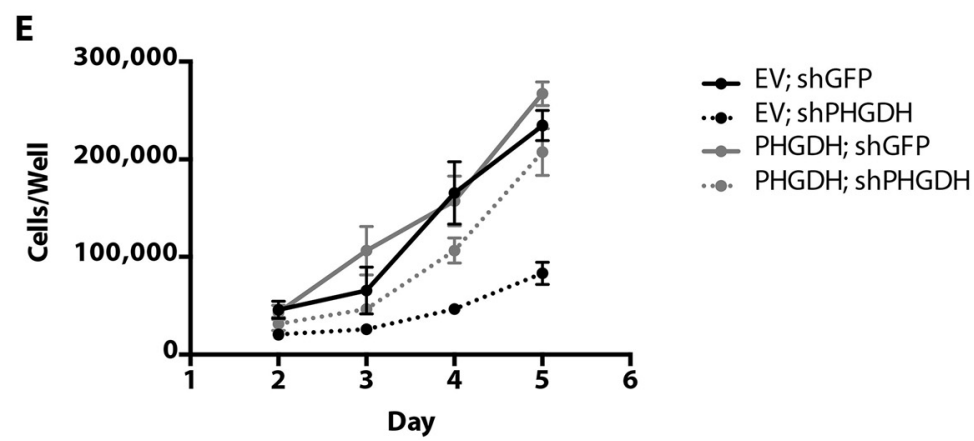

Figure $1 \mathrm{~N}$-terminal epitope-tagged PHGDH cannot support cell proliferation following PHGDH knockdown. (A) Schematic representation of the serine biosynthesis pathway. 3-PG, 3-phosphoglycerate; PHGDH, 3-phosphoglycerate dehydrogenase; NAD ${ }^{+} / \mathrm{NADH}$, oxidized and reduced forms of nicotinamide adenine dinucleotide, respectively; PHP, phosphohydroxypyruvate; PSAT, phosphoserine aminotransferase; Glu, glutamate; aKG, alphaketoglutarate; P-Ser, phosphoserine; PSPH, phosphoserine phosphatase; Ser, serine. (B) Cell number over time of PHGDH-amplified T.T. cells stably expressing either an shRNA-resistant FLAG-His-PHGDH cDNA or empty vector (EV) control. (C) Western blot analysis assessing knockdown of endogenous PHGDH and expression of FLAG-His-PHGDH cDNA. (D) Western blot analysis of T.T. cells stably expressing an shRNA-resistant PHGDH cDNA (untagged) or empty vector (EV) control. (E) Cell number over time of the cells described in (D) when infected with virus expressing GFP or PHGDH shRNA. Error bars show standard deviation from the mean.

synthesis and glutathione production [1]. In this way, the biosynthesis of serine from glucose plays a central role in cancer cell metabolism.

PHGDH expression is important in human cancers. PHGDH is located in a region of genomic copy number gain that includes four other genes in the smallest common amplified region [2]. PHGDH copy number gain has been observed with the highest frequency in melanoma and breast cancer, and $P H G D H$-amplified cell lines are dependent on enzyme expression to proliferate in culture $[3,4]$. Increased expression of PHGDH and other serine biosynthesis pathway enzymes is found in other cancer contexts as well, with evidence that c-Myc [5], ATF4 [6], and the lysine methyltransferase G9A [7] increase PHGDH expression via transcriptional and epigenetic mechanisms. Increased activity of serine biosynthesis pathway enzymes has been hypothesized to support increased nucleotide biosynthesis in cancer [8], although 
cell lines vary in their dependence on PHGDH expression for growth as xenograft tumors $[4,9]$.

Studies have also demonstrated that exogenous serine is critical for tumor cell proliferation in vitro and as xenografts [10-12]; however, there may be a distinct requirement for de novo serine synthesis from glucose even in the presence of abundant serine. Exogenous serine does not rescue proliferation of $P H G D H$-amplified cells following knockdown of PHGDH expression [4,9]; moreover, the expression of PSAT and PSPH is also required for the continued proliferation of at least some of these cells [3]. Taken together, these data suggest that flux through the serine biosynthesis pathway is important for cell metabolism beyond providing serine. One possibility is a role in TCA cycle anapleurosis [4], but it remains controversial whether this underlies the requirement for PHGDH in all cells $[2,3,13]$.

Interrogating PHGDH function(s) would benefit from studies of purified enzyme. Past comprehensive studies of PHGDH enzyme activity examined the Escherichia coli protein [14-17], which has a different domain structure than the human enzyme [18]. Structural studies of the human enzyme are limited to a truncated protein (PDB 2G76, unpublished), and enzymatic analysis often uses epitope-tagged protein $[19,20]$. While epitope tags are commonly used to isolate recombinant enzyme, in some cases, these tags can alter structure and/or function [21]. Therefore, both an understanding of whether an epitope tag will impact PHGDH function and methods to produce recombinant enzyme that reflects endogenous protein activity in cells are necessary to determine the role of PHGDH in human cancer and normal tissues.

We observed that, unlike untagged protein, the expression of PHGDH with an epitope tag was unable to rescue cell proliferation following PHGDH knockdown in PHGDH-amplified cells. An enzymatically dead PHGDH mutant also failed to rescue proliferation of cells following PHGDH knockdown, suggesting that enzyme activity is required for cells to proliferate. We present a method for purification of untagged recombinant PHGDH and find that the activity of this protein is appreciably greater than recombinant PHGDH with an N-terminal epitope tag. Finally, we show using size exclusion chromatography and electron microscopy that an $\mathrm{N}$-terminal epitope tag on PHGDH affects protein structure, which may contribute to an inability to substitute for endogenous PHGDH in cells. These data argue that the use of epitope tags should be avoided in future studies of PHGDH function.

\section{Methods}

\section{Cell culture, viral infection, and assessment of cell} proliferation

Cells were cultured in DMEM supplemented with 10\% FBS, penicillin, and streptomycin. We stably introduced
shRNA-resistant PHGDH cDNAs into cells using the retroviral vector pLHCX and selected with $150 \mu \mathrm{g} / \mathrm{ml}$ hygromycin. To decrease PHGDH expression, shRNA construct TRCN0000028548 with target sequence 5'-AGGTGATAA CACAGGGAACAT-3' was used. Cells were infected with lentivirus to express shRNAs, selected for 3 days in $1 \mu \mathrm{g} / \mathrm{ml}$ puromycin and proliferation assessed on subsequent days.

\section{Western blot analysis}

PHGDH protein was detected using a rabbit polyclonal anti-PHGDH antibody from Sigma, St. Louis, MO, USA (HPA021241) at $0.2 \mu \mathrm{g} / \mathrm{ml}$ and actin detected using a rabbit polyclonal anti-actin antibody from Abcam, Cambridge, MA, USA (ab1801) at $1 \mu \mathrm{g} / \mathrm{ml}$, appropriate HRP-conjugated secondary antibodies and chemiluminescence.

\section{Site-directed mutagenesis}

The PHGDH R236E mutant was generated using the QuickChange II XL Site-Directed Mutagenesis Kit from Agilent, Santa Clara, CA, USA, with the following primers: 5'-TGTGGTGAACTGTGCCGAGGGAGGGATCGTGGA CG-3' and 5' -CGTCCACGATCCCTCCCTCGGCACAGT TCACCACA-3'. To generate shRNA-resistant PHGDH, we used the same kit and the following primers: $5^{\prime}$-CCCAAA GGGACCATCCAAGTTATCACACAGGGAACATCCC3' and 5'-GGGATGTTCCCTGTGTGATAACTTGGATG GTCCCTTTGGG-3'.

\section{Purification of epitope-tagged PHGDH and PSAT proteins} pTriEx-3 FLAG-His-PHGDH (wild type or R236E mutant) or pET28a His-PHGDH was transformed into BL21 (DE3) E. coli. Single colonies from these plates were inoculated into LB plus ampicillin (pTriEx-3) or kanamycin (pET28a) and grown at $37^{\circ} \mathrm{C}$ and $225 \mathrm{rpm}$ overnight. The next day, cultures were diluted 1:40 and grown to $\mathrm{OD}_{600}=0.4$, and then $0.5 \mathrm{mM}$ IPTG was added before incubating at $16^{\circ} \mathrm{C}$ and $225 \mathrm{rpm}$ overnight. The bacteria were pelleted at $6,000 \times g$ for $15 \mathrm{~min}$, and the pellet was flash frozen and stored at $-80^{\circ} \mathrm{C}$. All protein purification steps were performed at $4^{\circ} \mathrm{C}$. To purify protein, bacterial pellets were resuspended in $30 \mathrm{ml}$ lysis buffer (50 mM Tris, $\mathrm{pH} 8.5,10$ $\mathrm{mM} \mathrm{MgCl}, 300 \mathrm{mM} \mathrm{NaCl}, 10 \%$ glycerol, and $5 \mathrm{mM}$ imidazole) and sonicated, and the lysate was centrifuged at $20,000 \times g$ for $45 \mathrm{~min}$. $\beta$-Mercaptoethanol $(30 \mu \mathrm{l})$ was added and the lysate mixed with $3 \mathrm{ml}$ of Ni-NTA agarose (Qiagen, Hilden, Germany) in wash buffer (50 mM Tris, $\mathrm{pH} 8.5,10$ $\mathrm{mM} \mathrm{MgCl}_{2}, 300 \mathrm{mM} \mathrm{NaCl}, 10 \%$ glycerol, and $30 \mathrm{mM}$ imidazole). This mixture was shaken gently for 2 to $3 \mathrm{~h}$, and Ni-NTA beads were pelleted and washed four times with wash buffer. The beads and lysate were transferred to an empty column and protein recovered in elution buffer (50 $\mathrm{mM}$ Tris, $\mathrm{pH}$ 8.5, $10 \mathrm{mM} \mathrm{MgCl}, 250 \mathrm{mM} \mathrm{NaCl}, 10 \%$ glycerol, and $250 \mathrm{mM}$ imidazole) with 1-ml fractions collected. Those fractions with high protein content were determined 
by Bradford assay. Fractions containing appreciable protein were pooled and separated on a GE HiPrep Sephacryl S-200 HR column (GE Healthcare Bio-Sciences, Pittsburgh, PA, USA) using an AKTA FPLC system (GE Healthcare Bio-Sciences, Pittsburgh, PA, USA). The column was run at $0.5 \mathrm{ml} / \mathrm{min}$ for one-column volume in storage buffer (20 $\mathrm{mM}$ Tris, pH 7.5, $100 \mathrm{mM} \mathrm{NaCl}, 1 \mathrm{mM}$ TCEP). Fractions were collected from the void volume to the elution volume of a $17-\mathrm{kDa}$ standard. UV absorbance was used to determine those fractions with the highest protein concentration, and those were pooled, $0.5 \mathrm{mM}$ betaine added, and concentrated to 5 to $10 \mu \mathrm{g} / \mu \mathrm{l}$ purified protein. Fractions from the size exclusion column were later analyzed by SDS-PAGE and Coomassie staining. After destaining, Coomassie-stained gels were imaged on a LiCOR and quantitated by densitometry analysis using ImageJ. Recombinant His-tagged PSAT was generated by an analogous procedure, with differences as follows. pET28a His-PSAT was transformed into BL21 (DE3) E. coli, grown as described above to an $\mathrm{OD}_{600}$ of 0.7 , then $0.5 \mathrm{mM}$ IPTG was added, and the culture was grown at room temperature and $225 \mathrm{rpm}$ for $6 \mathrm{~h}$. After rPSAT was eluted from the NiNTA agarose beads, the combined fractions were dialyzed into $50 \mathrm{mM}$ Tris, $\mathrm{pH} 7.5,10 \mathrm{mM} \mathrm{MgCl}_{2}, 25 \mathrm{mM} \mathrm{NaCl}$, $20 \%$ glycerol, $0.15 \%$ beta-mercaptoethanol.

\section{Preparation of Cibacron Blue F3GA Sepharose}

This protocol was modified from [22]. Sixty milliliters of GE Sepharose CL-4B resin (GE Healthcare Bio-Sciences, Pittsburgh, PA, USA) in water was mixed with $8.6 \mathrm{ml}$ of 45 $\mathrm{mg} / \mathrm{ml}$ Cibacron Blue F3GA (Polysciences, Warrington, PA, USA) for $30 \mathrm{~min}$ at room temperature. Twenty-seven milliliters of $2 \mathrm{M}$ sodium sulfate were added, followed by $30 \mathrm{~min}$ more mixing at room temperature. Two hundred ninety microliters of $10 \mathrm{M} \mathrm{NaOH}$ was added slowly, dropwise with mixing. The resulting bead suspension was mixed at $60^{\circ} \mathrm{C}$ for $2 \mathrm{~h}$ before neutralizing with $15 \mathrm{ml}$ of $2.5 \mathrm{M}$ sodium phosphate buffer, $\mathrm{pH} 7.5$. The beads were washed and then stored in $20 \%$ ethanol at $4{ }^{\circ} \mathrm{C}$ for at least 2 days. Two batches of beads were subsequently mixed and used to fill an empty GE XK 26/40 column (GE Healthcare Bio-Sciences, Pittsburgh, PA, USA) to a column volume of $65 \mathrm{ml}$ and attached to an AKTA FPLC system.

\section{Purification of untagged PHGDH protein}

pET28a PHGDH was transformed into BL21 E. coli. From a single colony, a culture was grown to an $\mathrm{OD}_{600}=0.4,0.5$ $\mathrm{mM}$ IPTG added, and the culture incubated at room temperature and $225 \mathrm{rpm}$ for $6 \mathrm{~h}$. The bacteria were flash frozen, thawed, and resuspended in $50 \mathrm{ml}$ buffer A (50 mM HEPES pH 8.0, 1 mM EDTA) plus $1 \mathrm{mM}$ DTT and protease inhibitors (Roche). This suspension was sonicated and centrifuged at $20,000 \times g$ for $45 \mathrm{~min}$, and the supernatant was loaded onto an F3GA column prepared as described above. The column was run at a flow rate of 2 $\mathrm{ml} / \mathrm{min}$ as follows: $200 \mathrm{ml}$ buffer $\mathrm{A}, 10-\mathrm{ml}$ linear gradient to 60\% buffer B (50 mM HEPES, pH 8.0, 1 mM EDTA, 400 $\mathrm{mM} \mathrm{KCl}), 130 \mathrm{ml} 60 \%$ buffer B. During the gradient and $60 \%$ B wash, 10-ml fractions were collected. Fractions were assessed for the presence of the recombinant protein by UV absorbance, pooled, concentrated, and desalted into buffer A. The resulting solution was fractionated on a GE MonoQ HR 10/10 column (GE Healthcare Bio-Sciences, Pittsburgh, PA, USA) using an AKTA FPLC at a flow rate of $0.5 \mathrm{ml} / \mathrm{min}$ as follows: $30 \mathrm{ml}$ buffer A, $45-\mathrm{ml}$ linear gradient to $45 \%$ buf-

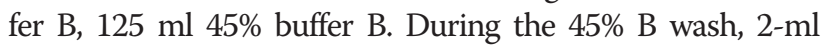
fractions were collected. Fractions were assessed for recombinant protein using UV absorbance, pooled and further separated using GE HiPrep Sephacryl S-200 HR column. The column fractions were treated and analyzed as described for epitope-tagged protein to produce the final protein solution.

\section{Assessment of PHGDH activity}

A linked assay was utilized where PHGDH, PSAT, and all substrates were mixed in a well of a 96-well plate and activity assessed at $37^{\circ} \mathrm{C}$. The reaction buffer used was $300 \mathrm{mM}$ Tris, pH 9, 1 mM EDTA, $1 \mathrm{mM}$ TCEP, 0.025\% BSA. A linked assay with PSAT was used to interrogate 3-PG and $\mathrm{NAD}+$ to PHP and NADH conversion by PHGDH because the equilibrium of this reaction lies toward 3-PG [23]. Enzyme activity was measured by following $\mathrm{NADH}$ generation over time by fluorescence, with excitation at $340 \mathrm{~nm}$ and emission at $460 \mathrm{~nm}$. All substrates were present in excess of their reported $K_{\mathrm{M}}$ values (final concentration $10 \mathrm{mM}$ each 3-PG, NAD ${ }^{+}$, and Glu). PSAT was present in excess of PHGDH and control experiments performed to ensure that PSAT was not limiting during the kinetic window evaluated.

\section{Immunofluorescence and confocal microscopy}

Cells were grown on glass coverslips and fixed with $4 \%$ paraformaldehyde. Cells were then permeabilized with PBS containing $0.1 \%$ Tween 20 , blocked in $1 \%$ BSA, and incubated in primary antibody overnight at $4{ }^{\circ} \mathrm{C}$. Primary antibody was rabbit polyclonal anti-PHGDH antibody from Sigma (HPA021241), used at $0.01 \mu \mathrm{g} / \mathrm{ml}$. Secondary antibody was AlexaFluor594 donkey anti-rabbit (A21207, Life Technologies, Grand Island, NY, USA) at $2 \mu \mathrm{g} / \mathrm{ml}$. Cells were counterstained with DAPI (D9542, Sigma, St. Louis, MO, USA) and mounted using Prolong Gold Antifade mounting medium (P36934, Life Technologies, Grand Island, NY, USA). Images were captured using a Deltavision Spectris imaging system (GE Healthcare BioSciences Corp., Piscataway, NJ, USA) with $\times 100$ oil immersion objective and analyzed using softWoRx (Applied Precision, Issaquah, WA, USA) and ImageJ (National Institutes of Health) software. Total fluorescence intensity was corrected using a secondary antibody-only control and staining of PHGDH knockout cell lines. 


\section{Mass spectrometry metabolite measurement}

Cells were extracted in ice cold 1:4:5 water:methanol:chloroform with valine-D8 as an internal standard. The aqueous layer was dried under $\mathrm{N}_{2}$ and analyzed by LC/ MS using a Q Exactive benchtop orbitrap mass spectrometer (Thermo Fisher Scientific, San Jose, CA, USA) equipped with a heated electrospray ionization (HESI) probe, coupled to a Dionex UltiMate 3000 UPLC system (Thermo Fisher Scientific, San Jose, CA, USA). For analysis, dried metabolites were resuspended in $100 \mu \mathrm{l}$ water, and $1 \mu \mathrm{l}$ of each sample was separated on a ZICpHILIC $2.1 \times 150 \mathrm{~mm}(5-\mu \mathrm{m}$ particle size $)$ column (EMD) at a flow rate of $0.150 \mathrm{ml} / \mathrm{min}$ as follows: 0 to 20 min: linear gradient from $80 \%$ to $20 \% \mathrm{~B}$; 20 to $20.5 \mathrm{~min}$ : linear gradient from $20 \%$ to $80 \% \mathrm{~B}$; and 20.5 to $28 \mathrm{~min}$ : hold at $80 \%$ B. Buffer A was 20 mM ammonium carbonate, $0.1 \%$ ammonium hydroxide; buffer $\mathrm{B}$ was acetonitrile. Metabolites were detected by mass spectrometry operating in full-scan, polarity switching mode with the spray voltage set to $3.0 \mathrm{kV}$, the heated capillary held at $275^{\circ} \mathrm{C}$, and the HESI probe held at $350^{\circ} \mathrm{C}$. The sheath gas flow was set to 40 units, the auxiliary gas flow was set to 15 units, and the sweep gas flow was set to 1 unit. The MS data acquisition was performed in a range of 70 to 1,000 $\mathrm{m} / z$, with the resolution set at 70,000, the AGC target at $10^{6}$, and the maximum injection time at $80 \mathrm{~ms}$. Relative quantitation of polar metabolites was performed with XCalibur QuanBrowser 2.2 (Thermo Fisher Scientific, San Jose, CA, USA) using a 5 ppm mass tolerance and referencing an in-house library of chemical standards.

\section{Size exclusion chromatography}

Either recombinant His-PHGDH or recombinant untagged PHGDH was analyzed using a GE HiPrep Sephacryl S-200 HR column. The column was run at $0.5 \mathrm{ml} /$ min for one-column volume in storage buffer. Fractions were collected from just before the void volume to the elution volume of a $17-\mathrm{kDa}$ standard. Fractions were then subjected to SDS-PAGE, and the gels were stained with colloidal Coomassie. After destaining, the gel was imaged and quantitated as described above.

\section{Electron microscopy and image processing}

Specimens were prepared using $12 \mathrm{ng} / \mu \mathrm{l} \mathrm{His-PHGDH}$ and $15 \mathrm{ng} / \mu \mathrm{l}$ untagged PHGDH with $0.5 \mathrm{mM}$ NADH by adsorbing $5 \mu$ l for approximately $1 \mathrm{~min}$ to carbon-coated EM grids that had been glow discharged immediately before use, negative staining with $2 \%$ uranyl acetate, sandwiching under a second layer of carbon film, blotting, and drying [24]. On an FEI Tecnai Spirit BioTwin microscope (FEI Tecnai, Hillsboro, OR, USA) with a tungsten filament and an AMT XR16 CCD detector, 103 images of the untagged PHGDH preparation and 107 images of the His-PHGDH preparation were acquired at 0.5 - to
1.0- $\mu \mathrm{m}$ defocus (assessed using CTFFIND3 [25] implemented in SPIDER [26]). Identical microscope and camera settings were used for imaging both specimens: $\times 60,000$ magnification (1.84 $\AA$ /pixel at the specimen level), $80-\mathrm{kV}$ acceleration voltage, and 1.6-s exposure.

Particles were selected with e2boxer from eightfold binned, band-pass-filtered images. The 23,568 PHGDH particles and 30,894 His-PHGDH particles were cut from the original fullsized images into windows of $160 \times 160$ pixels using EMAN2 [27]. In preparation for processing, particle images were high-pass filtered, outlier densities median filtered, downsampled fivefold to 32-pixel squares of $9.20 \AA$ A/pixel, and normalized such that background densities were scaled to $0 \pm 1$. Particles from each dataset were aligned, clustered, and realigned within clusters with SPARX using identical reference-free alignment (sxali2d) and k-means clustering (sxkmeans) parameters [28]. To sort averages according to particle size, averages were binarized at a threshold (0.25) that distinguishes the background gray values from the foreground particle densities. Distributions of particle sizes were plotted with Gnuplot. Kolmogorov-Smirnov statistical tests for size distributions of the two samples were calculated using the stats module of SciPy and corroborated with Prism (GraphPad Software).

\section{Results}

Untagged PHGDH, but not N-terminally tagged PHGDH, can rescue cell proliferation following PHGDH knockdown Knockdown of PHGDH expression using any of several different small hairpin RNAs impairs proliferation of PHGDH-amplified cell lines [3,4]. However, even when multiple hairpins are used, off-target effects can complicate interpretation of results from RNA interference experiments [29]. To confirm that loss of PHGDH expression decreases proliferation of PHGDH-amplified cells, we introduced silent mutations into the PHGDH cDNA to render it resistant to shRNA-mediated knockdown. Initially, an N-terminal FLAG-His epitope tag was included for ease of protein detection and purification. To determine whether FLAG-HisPHGDH expression can rescue shRNA-mediated knockdown of endogenous PHGDH expression, T.T. cells were engineered to express either shRNA-resistant PHGDH cDNA or empty vector as a control. Proliferation of T.T. cells, an esophageal cancer cell line with $P H G D H$ copy number gain, has been shown previously to be sensitive to stable knockdown of PHGDH expression [3]. Consistent with previous studies, stable knockdown of $\mathrm{PHGDH}$ in control T.T. cells impaired proliferation (Figure 1B). However, exogenous expression of tagged PHGDH failed to rescue this decrease in cell proliferation despite the presence of tagged PHGDH protein (Figure 1B,C). To determine whether exogenously expressed PHGDH failed to rescue proliferation because the epitope tag interfered with protein function, T.T. cells were engineered to express shRNA- 
resistant PHGDH cDNA lacking an epitope tag (Figure 1D). Expression of PHGDH without an epitope tag rescued proliferation following knockdown of PHGDH (Figure 1E), in agreement with findings in other cells [9]. These findings suggest that introduction of an N-terminal epitope tag impairs the ability of PHGDH to support T.T. cell proliferation.

\section{PHGDH enzymatic activity is required to rescue cell} proliferation following PHGDH knockdown

Despite an inability to rescue proliferation, recombinant FLAG-His-PHGDH exhibits enzyme activity in vitro (Figure 2A). PHGDH enzyme activity was measured by tracking $\mathrm{NADH}$ production when $\mathrm{NAD}^{+}$and 3-PG were provided at saturating concentrations, with the product PHP removed by coupling the assay to PSAT and providing glutamate (Figure 1A). To confirm that the ability of exogenous PHGDH to rescue endogenous PHGDH knockdown is dependent on enzymatic activity, we generated a mutant that was predicted to be enzymatically inactive. The positively charged arginine residue at position 236 of PHGDH is thought to anchor the carboxyl group of 3-PG. R236 in human PHGDH is analogous to R240 in E. coli PHGDH, which when replaced with an alanine was effective at disrupting bacterial enzyme function [16]. Thus, we determined whether mutation of R236 in human PHGDH to a negatively charged glutamate would disrupt enzyme function. FLAG-His-PHGDH R236E was purified from E. coli and had no enzyme activity in vitro (Figure $2 \mathrm{~A}$ ). We stably overexpressed an shRNA-resistant, untagged version of the R236E mutant in T.T. cells and found that it was incapable of rescuing cell proliferation following knockdown of endogenous PHGDH (Figure 2B and Additional file 1: Figure S1). These data support PHGDH enzymatic function being required for the proliferation of T.T. cells.

\section{Purification of untagged PHGDH}

To study the differences between tagged and untagged PHGDH in vitro, we developed a method to purify the untagged protein. Dye-based pseudoaffinity ligands have been useful for purification of many other enzymes [30,31]. An evaluation of dye-based ligands that bound other enzymes that use $\mathrm{NAD}(\mathrm{H})$ as a co-substrate identified Cibacron Blue F3GA as a pseudoaffinity ligand capable of enriching PHGDH protein expressed in E. coli from other proteins by severalfold (Figure 3A). We then designed a purification

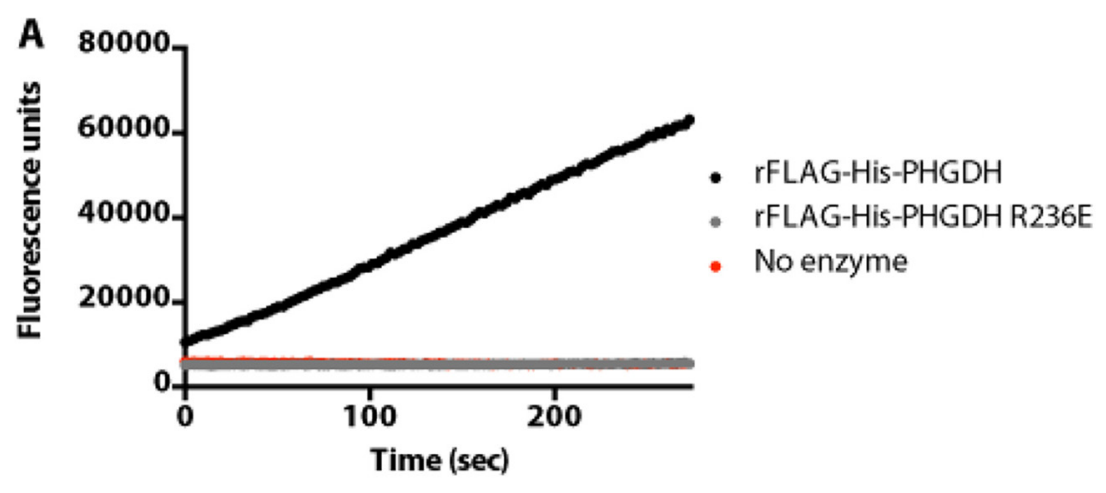

B
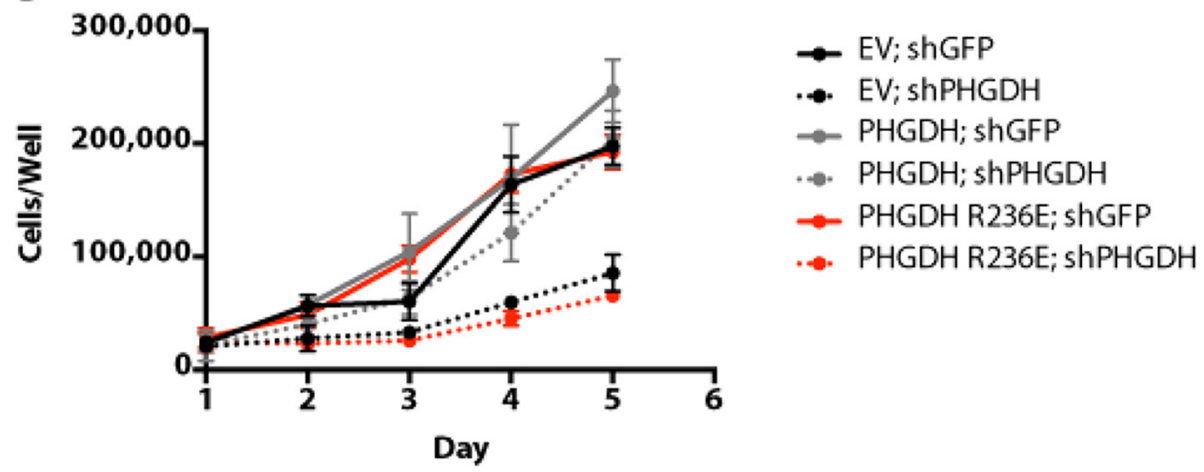

Figure 2 PHGDH enzymatic activity is required for cell proliferation following PHGDH knockdown. (A) In vitro enzyme activity assessed by tracking NADH production by fluorescence. The assay was performed at saturating substrate concentrations. (B) Cell number over time for PHGDH-amplified T.T. cells stably expressing shRNA-resistant PHGDH wild type or R236E (enzymatically dead) cDNAs or empty vector (EV) control when infected with virus expressing GFP or PHGDH shRNA. Error bars show standard deviation from the mean. 
A

B
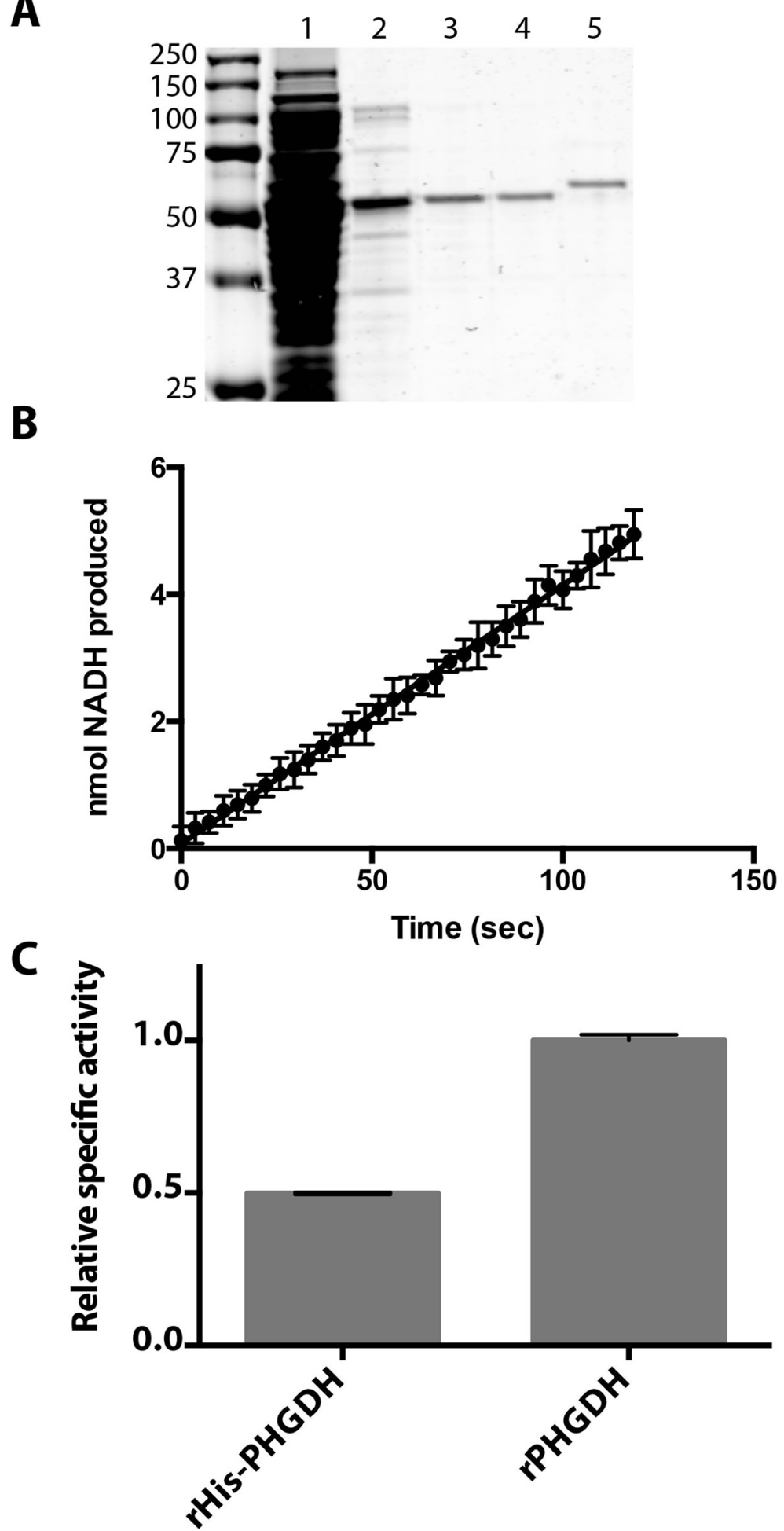

Figure 3 Purified untagged recombinant PHGDH has higher specific activity than N-terminally tagged PHGDH. (A) Coomassie gel of rPHGDH (untagged) after each step of the purification: 1. cleared lysate from E. coli expressing rPHGDH protein, 2. eluate from Cibacron Blue F3GA column, 3. eluate from anion exchange column, 4. eluate from size exclusion column, and 5. rFLAG-His-PHGDH purified by sequential nickel and size exclusion columns, for comparison. Molecular weight standards in kilodaltons are present to the left of lane 1. (B) In vitro enzyme activity of untagged rPHGDH. Assay was performed at saturating substrate concentrations. (C) Comparison of relative specific activity of rPHGDH and rHis-PHGDH. Error bars show standard deviation from the mean. 
scheme involving three sequential chromatography steps: affinity chromatography using F3GA binding, ion exchange chromatography, and size exclusion chromatography. Using this approach, we recover E. coli-expressed PHGDH protein to the same approximate level of purity as His-FLAG-tagged PHGDH prepared using Ni-based affinity chromatography and size exclusion chromatography (Figure 3A).

\section{Untagged recombinant PHGDH has higher specific activity than $\mathrm{N}$-terminally tagged PHGDH}

Untagged recombinant protein is active and has linear kinetics in an in vitro assay. The absolute specific activity of untagged PHGDH under these conditions is $0.071 \pm 0.001$ $\mathrm{nmol} \mathrm{NADH}$ produced/s/ $\mu \mathrm{g}$ protein (Figure $3 \mathrm{~B}$ ). This specific activity is twice the specific activity of recombinant HisPHGDH (Figure 3C), which again exhibits about twice the relative specific activity of recombinant FLAG-His-PHGDH (Additional file 2: Figures S2A, B).

\section{$\mathrm{N}$-terminally tagged and untagged PHGDH display the} same intracellular localization

To determine if the epitope tag disrupts PHGDH localization, MDA-MB-231 cells, which express very little PHGDH [4], and PHGDH-amplified T.T. cells were engineered to express vector alone, untagged PHGDH, or FLAG-His-PHGDH and the spatial distribution of PHGDH determined by immunofluorescence. As expected, MDA-MB-231 cells expressing empty vector control showed very little PHGDH staining. In all other samples, PHGDH showed widespread cytoplasmic staining with no difference observed when epitope-tagged PHGDH was introduced (Figure 4A,B). These data are consistent with $\mathrm{N}$-terminally tagging PHGDH having no effect on enzyme localization and argue that an effect on localization does not explain the inability of tagged PHGDH to support cell proliferation.

\section{Cells expressing $\mathrm{N}$-terminally tagged and untagged PHGDH have an equal 2HG:aKG ratio}

In addition to catalyzing 3-PG conversion to PHP, PHGDH from both $E$. coli and humans can also produce D-2hydroxyglutarate (D-2HG) as a minor product [13,32]. Because D-2HG has been implicated in the pathogenesis of some human cancers we examined whether N-terminally tagged PHGDH results in lower 2HG production than wildtype PHGDH in cells. We measured the 2HG: $\alpha$ KG ratio of T.T. and MDA-MB-231 cells expressing either Nterminally tagged or untagged PHGDH by LC/MS. In both cell lines, 2HG: $\alpha$ KG ratios were not significantly different regardless of which enzyme was expressed (Additional file 3: Figure S3), suggesting that $\mathrm{N}$ terminally tagged PHGDH produces the same amount of $2 \mathrm{HG}$ as untagged PHGDH in cells.
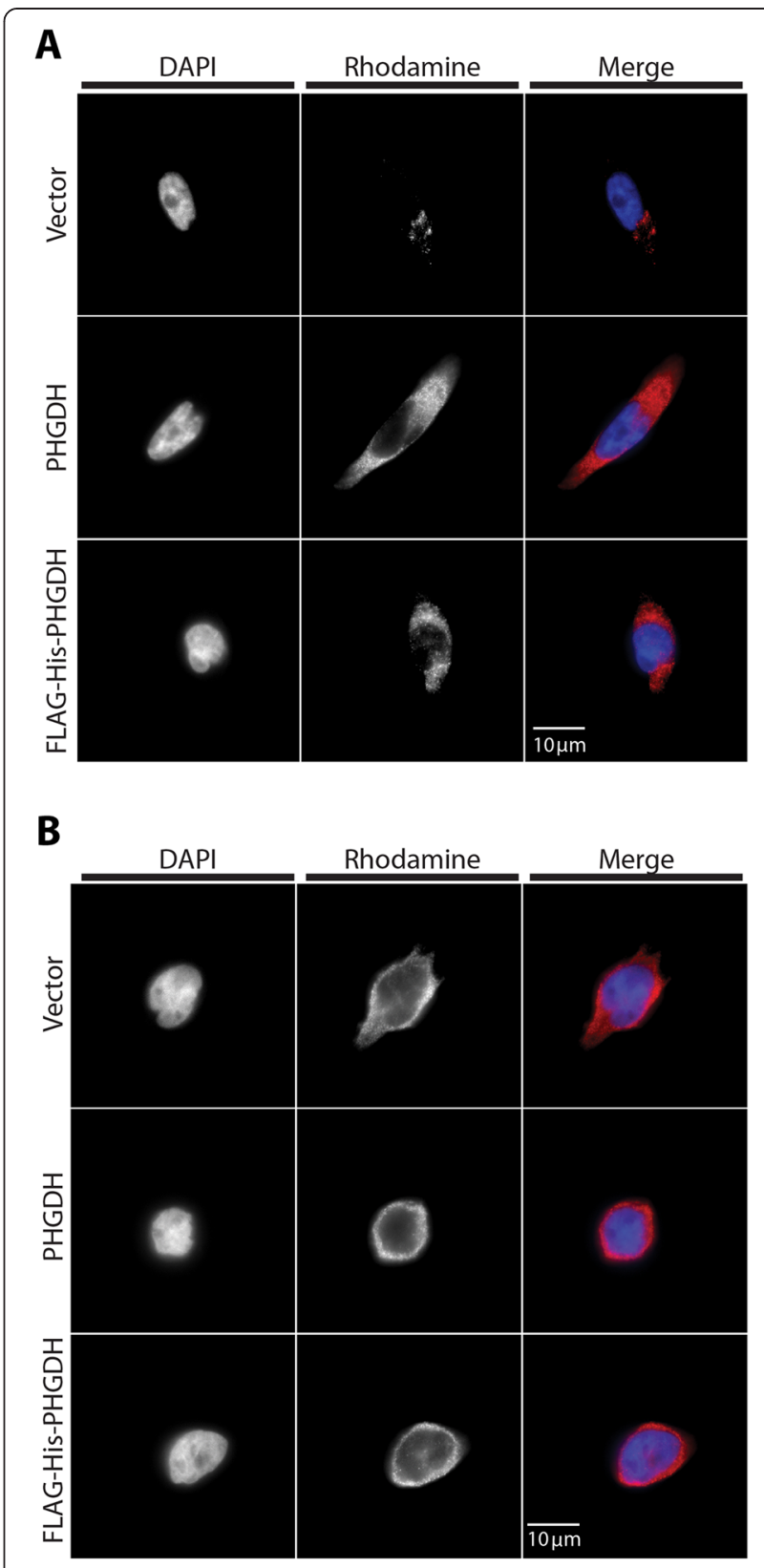

Figure $4 \mathrm{~N}$-terminal epitope-tagged and untagged PHGDH display the same intracellular localization. (A) MDA-MB-231 cells, which are PHGDH low, and (B) PHGDH-amplified T.T. cells expressing either vector control, untagged, or $\mathrm{N}$-terminally tagged $\mathrm{PHGDH}$ were stained for PHGDH immunofluorescence.

$\mathrm{N}$-terminally tagged and untagged PHGDH exhibit different structural properties by size exclusion analysis To interrogate structural differences that might exist between the tagged and untagged recombinant protein, we assessed the mobility of each by size exclusion chromatography. We found that the elution volume for tagged versus untagged PHGDH differed appreciably, with rHis-PHGDH eluting much closer to the void volume of a Sephacryl S-200 HR column (GE Healthcare Bio- 
Sciences, Pittsburgh, PA, USA), which can resolve globular proteins from 5 to $250 \mathrm{kDa}$ (Figure 5). Comparison with standards of known molecular mass indicates that the peak of untagged PHGDH is approximately $155 \mathrm{kDa}$, whereas the peak of His-PHGDH is approximately 240 $\mathrm{kDa}$. These data argue that an N-terminal tag affects the mobility of PHGDH on a size exclusion column, and this change in structure may affect the ability of the enzyme to support cell proliferation in some cancer cells.

\section{$\mathrm{N}$-terminally tagged PHGDH forms larger macromolecular complexes than untagged PHGDH}

To further evaluate potential differences in the structure of the tagged and untagged recombinant protein, negatively stained specimens of each purified protein were prepared and imaged by transmission electron microscopy and protein particles were analyzed by standard methods for alignment and clustering. Comparing the class averages from the PHGDH and His-PHGDH specimens suggests that

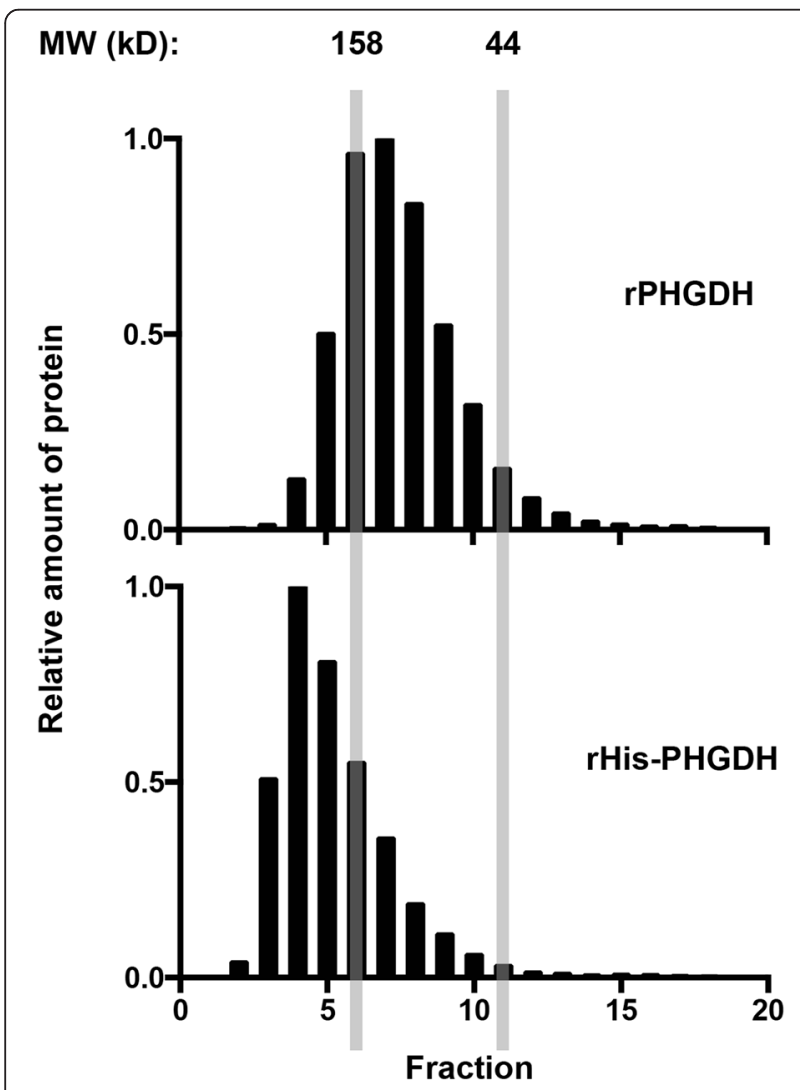

Figure $5 \mathrm{~N}$-terminal epitope-tagged and untagged PHGDH exhibit different mobility on size exclusion chromatography. Relative amount of $r P H G D H$ and rHis-PHGDH protein present in fractions eluted from a Sephacryl S-200 size exclusion column. The 158- and 44-kDa standards are shown for comparison, with the column void volume represented in fraction 2 . both preparations have overlapping macromolecular sizes and shapes (Figure 6A,B). To quantitatively assess whether the distribution of particle sizes are different, the area of each average was calculated and compared between PHGDH and His-PHGDH (Figure 6C). The His-PHGDH particle size distribution is significantly shifted toward more massive species $(P$ value $<0.0001)$ with a substantial population of particles of size greater than $11,000 \AA^{2}$. The altered distribution is reflected in increased mean particle size of His-PHGDH $\left(8,922 \AA^{2}\right)$ relative to PHGDH $\left(7,855 \AA^{2}\right)$. Given the estimated masses of the complex from size exclusion chromatography, these areas are about twice what would be expected for a dimer, trimer, or tetramer, suggesting that the oligomeric structures of human PHGDH are not approximated well by a sphere. This data demonstrates directly that an N-terminal epitope tag affects the PHGDH structure, which may in turn influence the activity of the protein in vitro and the ability to support proliferation after knockdown of the endogenous enzyme.

\section{Discussion}

We demonstrate that N-terminally tagged PHGDH is not functionally equivalent to endogenous PHGDH. Epitope tags have been used for more than 25 years [33-36] and are useful for both purification of recombinant proteins and protein detection when antibodies to an endogenous protein are not available. Although many tags are well tolerated [21], introducing an exogenous peptide sequence onto the $\mathrm{N}$-terminus of PHGDH disrupts protein structure and function. High-quality antibodies for PHGDH are available, and a scheme for PHGDH purification using commercially available materials is described here that renders the use of epitope tags on PHGDH unnecessary.

$\mathrm{N}$-terminally tagged PHGDH not only retains some enzymatic activity in vitro but also exhibits structural differences from the endogenous protein; understanding how the tag disrupts PHGDH structure and function could provide new insight into how this protein supports cell proliferation. There is no atomic-resolution structure of the full-length human PHGDH protein, and more detailed structural information would aid in understanding why an $\mathrm{N}$-terminal tag perturbs PHGDH function. PHGDH enzymes from various organisms are grouped into three types (types I to III) based on differences in C-terminal domains thought to be involved in enzyme regulation [37]. All type I and II PHGDH enzymes examined, including E. coli [17], M. tuberculosis [38], chicken [15], rat [39], and rabbit [40] form tetramers. The inter-subunit interfaces in both type I and II enzymes contain contacts involving the C-terminal regulatory domains. As a result, type III enzymes that lack a Cterminal domain form dimers [37,38]. Human PHGDH is 

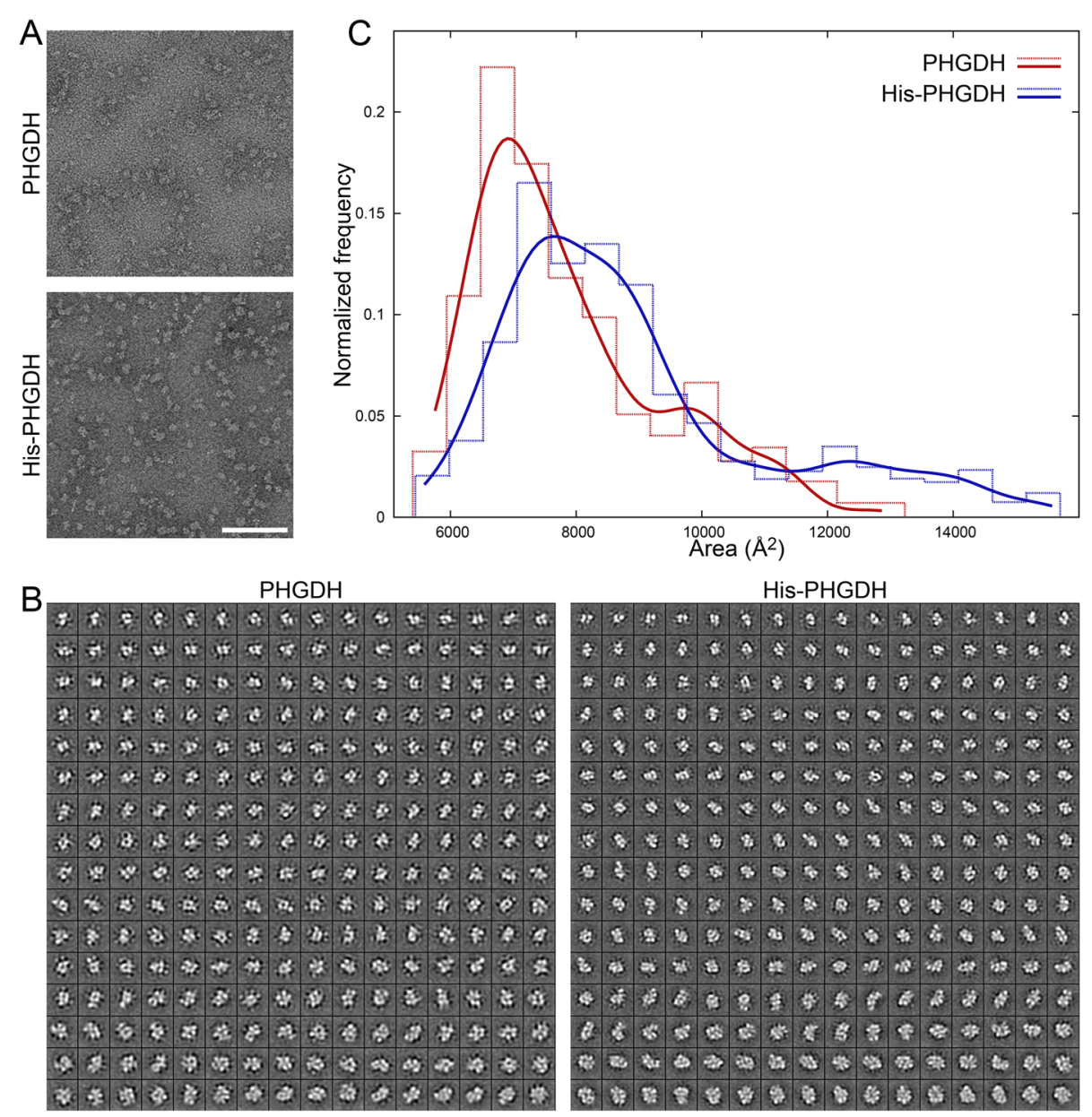

Figure 6 N-terminally tagged PHGDH forms larger macromolecular complexes than untagged PHGDH. (A) Representative images obtained from PHGDH and His-PHGDH specimens. Scale bar $=100 \mathrm{~nm}$. (B) Montage of class averages of PHGDH and His-PHGDH sorted from smallest (top row) to largest (bottom row). Each average is $294 \AA$ wide at the specimen level. (C) Normalized particle-size distributions (particles per bin/total particles) are displayed as histograms (dashed lines) and as smoothed curves (solid lines) for PHGDH (blue) and His-PHGDH (red). The His-PHGDH and PHGDH histograms are offset by $42 \AA^{2}$ to avoid overlapping lines.

a type I enzyme and is therefore predicted to function as a tetramer; however, the only human PHGDH structure is of a truncated protein lacking the C-terminal domains and thus crystallized as a dimer (PDB 2G76, unpublished). Despite dimeric type III enzymes retaining catalytic function, all plant and animal PHGDH enzymes characterized so far are type I $[18,41]$, suggesting that the C-terminal regulatory domains are important for PHGDH function in these cells.

Few full-length type I and II PHGDH protein structures have been solved; however, examples of both are available. The E. coli protein is an example of a type II $\mathrm{PHGDH}$ with a known structure and is a symmetrical dimer of dimers in which all monomers assume an identical configuration. In E. coli PHGDH, one dimer interface is formed by two adjacent nucleotide binding domains and the other is formed by two C-terminal regulatory domains [17] (PDB 1YBA). The N-terminus of this PHGDH protein appears to be in a position that would accommodate an epitope tag without disrupting the core protein structure. Mycobacterium tuberculosis $\mathrm{PHGDH}$ is an example of a type I protein [38] (PDB $1 Y G Y)$. In contrast to the E. coli protein, M. tuberculosis $\mathrm{PHGDH}$ is a tetramer formed by PHGDH monomers with identical amino acid sequence but two different tertiary structures. In the two monomer conformations, the regulatory domains are rotated by $180^{\circ}$. This results in inter-subunit interfaces that involve more complex inter-subunit contacts than the E. coli structure [38]. Human PHGDH is also a type I protein, suggesting that its structure may be more similar to $M$. tuberculosis than E. coli. In the M. tuberculosis structure, the Nterminus is not directly involved in any of the subunitsubunit interfaces, but the increased complexity of the 
interfaces may account for why placing a tag in this position on the human protein affects structure and function.

There remain a few possibilities for what PHGDH function is disrupted by the N-terminal tag. A threshold of PHGDH activity may be required to support proliferation, and despite retaining canonical enzyme activity, the untagged version is less active and may not be able to attain a level of activity required by proliferating cells. Alternatively, PHGDH could have a non-canonical activity that has not been fully appreciated. The only known PHGDH enzyme activity outside of serine synthesis is an ability to generate small amounts of D-2HG. Because $\mathrm{N}$-terminally tagged PHGDH generates the same 2HG: $\alpha$ KG ratio as untagged PHGDH, it is unlikely that a difference in 2HG production is responsible for the inability of tagged enzyme to support proliferation. Furthermore, the inability of tagged PHGDH to support cell proliferation despite production of similar 2HG levels as the wild-type enzyme, even in MDA-MB-231 cells, which express little to no endogenous PHGDH, also argues against $2 \mathrm{HG}$ production as the sole explanation for how increased PHGDH contributes to cancer. If another unknown activity of PHGDH is necessary to support proliferation, our results imply that it must also be lost in the R236E mutant.

PHGDH has been proposed as a potential drug target in cancer [42], and the impact of an epitope tag has practical implications for designing screens for small-molecule PHGDH inhibitors. Because tagged PHGDH retains some enzyme activity, the convenience of generating recombinant tagged protein might result in the use of this protein for drug screening. Whether this will identify molecules that impair proliferation of PHGDH-dependent cells remains to be determined, but the use of recombinant untagged protein is preferred based on our findings. If small molecules are identified using either approach, they could provide insight into whether the ability to inhibit 3-PG to PHP conversion alone is the relevant PHGDH function in cells and help understand the role of this enzyme in cell proliferation and human cancer.

\section{Conclusions}

Addition of an N-terminal epitope tag to PHGDH alters enzyme structure, reduces catalytic activity, and abrogates its ability to support proliferation of PHGDH-dependent cancer cells. A simple and effective scheme for purification of recombinant untagged PHGDH is presented, making use of epitope tags to generate purified enzyme unnecessary.

\section{Additional files}

Additional file 1: Figure S1. Western blot analysis of $\mathrm{PHGDH}$ expression in the T.T. cells shown in Figure 2B.
Additional file 2: Figure S2. In vitro enzyme activity and relative specific activity of rHis-PHGDH and rFLAG-His-PHGDH. (A) In vitro enzyme activity of rHis-PHGDH and rFLAG-His-PHGDH was assessed as in Figure 2A. (B) Comparison of relative specific activity of $r$ His-PHGDH and rFLAG-His$\mathrm{PHGDH}$. Error bars show standard deviation from the mean.

Additional file 3: Figure S3. Relative intracellular $2 \mathrm{HG}$ :aKG ratio as measured by LC/MS. Error bars show standard error of the mean. $P$ values were calculated by a two-tailed Student's t-test.

\section{Abbreviations}

3-PG: 3-phosphoglycerate; D-2HG: D-2-hydroxyglutarate; IMAC: immobilized metal ion affinity chromatography; PHGDH: phosphoglycerate dehydrogenase; PHP: phosphohydroxypyruvate; PSAT: phosphoserine aminotransferase; P-Ser: phosphoserine; PSPH: phosphoserine phosphatase; SHMT: serine hydroxymethyltransferase; TCA cycle: tricarboxylic acid cycle; aKG: alpha-ketoglutarate.

\section{Competing interests}

MGVH is a consultant and scientific advisory board member for Agios Pharmaceuticals. The remaining authors have no competing interests.

\section{Authors' contributions}

KRM and MGVH designed the study. KRM performed all the experiments other than the electron microscopy. EJB and CLD collected and analyzed the electron microscopy data. MK and BPF developed the protocol for making the F3GA resin. SMD collected and analyzed the immunofluorescence data. KRM and MGVH wrote the paper. All authors read and approved the final manuscript.

\section{Acknowledgements}

We acknowledge the support from the NSF GRFP DGE-1122374, T32GM007287, and the Paul Schimmel Graduate Student Fellowship, as well as funding from the NCl (R01CA168653, P30CA14051), the Burroughs Wellcome Fund, and the American Association for Cancer Research. We thank Elizaveta Freinkman and the Whitehead Institute Metabolite Profiling Core Facility for the assistance with LC/MS. We also thank Hadar Sharfi, Atsuo Sasaki, Jason Locasale, Lewis Cantley, Michael Pacold, and David Sabatini for sharing DNA constructs. Electron microscopy was performed at the W.M. Keck Microscopy Facility at the Whitehead Institute. CLD is a Howard Hughes Medical Institute Investigator.

\section{Author details}

${ }^{1}$ Koch Institute for Integrative Cancer Research, Massachusetts Institute of Technology, Cambridge, MA 02139, USA. ²Department of Biology, Massachusetts Institute of Technology, Cambridge, MA 02139, USA. ${ }^{3}$ Department of Chemistry Massachusetts Institute of Technology, Cambridge, MA 02139, USA. ${ }^{4}$ Howard Hughes Medical Institute Massachusetts Institute of Technology, Cambridge, MA 02139, USA. ${ }^{5}$ Dana-Farber Cancer Institute, Boston, MA 02215, USA. ${ }^{6}$ Broad Institute, Cambridge, MA 02139, USA.

Received: 17 November 2014 Accepted: 31 March 2015

Published online: 29 April 2015

\section{References}

1. Locasale JW. Serine, glycine and one-carbon units: cancer metabolism in full circle. Nat Rev Cancer. 2013;13:572.

2. Mullarky E, Mattaini KR, Vander Heiden MG, Cantley LC, Locasale JW. PHGDH amplification and altered glucose metabolism in human melanoma. Pigment Cell Melanoma Res. 2011;24:1112.

3. Locasale JW, Grassian AR, Melman T, Lyssiotis CA, Mattaini KR, Bass AJ, et al. Phosphoglycerate dehydrogenase diverts glycolytic flux and contributes to oncogenesis. Nat Genet. 2011;43:869.

4. Possemato R, Marks KM, Shaul YD, Pacold ME, Kim D, Birsoy K, et al. Functional genomics reveal that the serine synthesis pathway is essential in breast cancer. Nature. 2011:476:346.

5. Nilsson LM, Forshell TZP, Rimpi S, Kreutzer C, Pretsch W, Bornkamm GW, et al. Mouse genetics suggests cell-context dependency for Myc-regulated metabolic enzymes during tumorigenesis. PLoS Genet. 2012;8:e1002573.

6. Adams CM. Role of the transcription factor ATF4 in the anabolic actions of insulin and the anti-anabolic actions of glucocorticoids. J Biol Chem. 2007;282:16744. 
7. Ding J, Li T, Wang $X$, Zhao E, Choi JH, Yang L, et al. The histone H3 methyltransferase G9A epigenetically activates the serine-glycine synthesis pathway to sustain cancer cell survival and proliferation. Cell Metab. 2013;18:896.

8. Snell K, Natsumeda Y, Eble JN, Glover JL, Weber G. Enzymic imbalance in serine metabolism in human colon carcinoma and rat sarcoma. $\mathrm{Br} J$ Cancer. 1988;57:87.

9. Chen J, Chung F, Yang G, Pu M, Gao H, Jiang W, et al. Phosphoglycerate dehydrogenase is dispensable for breast tumor maintenance and growth. Oncotarget. 2013:4:2502

10. Labuschagne CF, van den Broek NJ, Mackay GM, Vousden KH, Maddocks $\mathrm{OD}$. Serine, but not glycine, supports one-carbon metabolism and proliferation of cancer cells. Cell Rep. 2014;7:1248.

11. Maddocks OD, Berkers CR, Mason SM, Zheng L, Blyth K, Gottlieb E, et al. Serine starvation induces stress and p53-dependent metabolic remodelling in cancer cells. Nature. 2013;493:542.

12. Kung C, Hixon J, Choe S, Marks K, Gross S, Murphy E, et al. Small molecule activation of PKM2 in cancer cells induces serine auxotrophy. Chemistry Biol. 2012;19:1187.

13. Fan JT, Xin LL, Mattaini K, Looper R, Vander Heiden M, Rabinowitz, J. Human phosphoglycerate dehydrogenase produces the oncometabolite D-2hydroxyglutarate. ACS Chem Biol. 2015;10:510.

14. Al-Rabiee R, Lee EJ, Grant GA. The mechanism of velocity modulated allosteric regulation in D-3-phosphoglycerate dehydrogenase: cross-linking adjacent regulatory domains with engineered disulfides mimics effector binding. J Biol Chem. 1996;271:13013.

15. Grant GA, Bradshaw RA. D-3-phosphoglycerate dehydrogenase from chicken liver. II. Chemical and physical properties. J Biol Chem. 1978;253:2727.

16. Grant GA, Kim SJ, Xu XL, Hu Z. The contribution of adjacent subunits to the active sites of D-3-phosphoglycerate dehydrogenase. J Biol Chem. 1999;274:5357.

17. Thompson JR, Bell JK, Bratt J, Grant GA, Banaszak L. Vmax regulation through domain and subunit changes: the active form of phosphoglycerate dehydrogenase. Biochemistry. 2005;44:5763.

18. Dey S, Hu Z, Xu XL, Sacchettini JC, Grant GA. D-3-phosphoglycerate dehydrogenase from Mycobacterium tuberculosis is a link between the Escherichia coli and mammalian enzymes. J Biol Chem. 2005;280:14884.

19. Tabatabaie L, de Koning TJ, Geboers AJ, van den Berg IE, Berger R, Klomp LW, et al. Novel mutations in 3-phosphoglycerate dehydrogenase (PHGDH) are distributed throughout the protein and result in altered enzyme kinetics. Hum Mutat. 2009;30:749.

20. Hitosugi T, Zhou L, Elf S, Fan J, Kang HB, Seo JH, et al. Phosphoglycerate mutase 1 coordinates glycolysis and biosynthesis to promote tumor growth. Cancer Cell. 2012;22:585.

21. Jarvik JW, Telmer CA. Epitope tagging. Annu Rev Genet. 1998;32:601.

22. Bohme HJ, Kopperschlager G, Schulz J, Hofmann E. Affinity chromatography of phosphofructokinase using Cibacron blue F3G-A. J Chromatogr. 1972;69:209.

23. Snell K. Enzymes of serine metabolism in normal, developing and neoplastic rat tissues. Adv Enzym Regul. 1984;22:325.

24. Tischendorf GW, Zeichhardt H, Stoffler G. Location of proteins S5, S13 and S14 on the surface of the 30 S ribosomal subunit from Escherichia coli as determined by immune electron microscopy. Mol General Genetics. 1974;134:209.

25. Mindell JA, Grigorieff N. Accurate determination of local defocus and specimen tilt in electron microscopy. J Struct Biol. 2003;142:334.

26. Frank J, Radermacher M, Penczek P, Zhu J, Li Y, Ladjadj M, et al. SPIDER and WEB: processing and visualization of images in 3D electron microscopy and related fields. J Struct Biol. 1996;116:190.

27. Tang G, Peng L, Baldwin PR, Mann DS, Jiang W, Rees I, et al. EMAN2: an extensible image processing suite for electron microscopy. J Struct Biol. 2007;157:38.

28. Hohn M, Tang G, Goodyear G, Baldwin PR, Huang Z, Penczek PA, et al. SPARX, a new environment for Cryo-EM image processing. J Struct Biol. 2007;157:47

29. Jackson AL, Bartz SR, Schelter J, Kobayashi SV, Burchard J, Mao M, et al. Expression profiling reveals off-target gene regulation by RNAi. Nat Biotechnol. 2003;21:635.

30. Labrou NE, Karagouni A, Clonis YD. Biomimetic-dye affinity adsorbents for enzyme purification: application to the one-step purification of Candida boidinii formate dehydrogenase. Biotechnol Bioeng. 1995;48:278.
31. Tulsani NB, Kumar A, Vijayalakshmi MA. Purification of muscle enzymes by pseudoaffinity chromatography. Preparative Biochemis Biotechnol. 1999;29:151.

32. Zhao G, Winkler ME. A novel alpha-ketoglutarate reductase activity of the serA-encoded 3-phosphoglycerate dehydrogenase of Escherichia coli K-12 and its possible implications for human 2-hydroxyglutaric aciduria. J Bacteriol. 1996;178:232.

33. Hopp TP, Prickett KS, Price VL, Libby RT, March CJ, Cerretti DP, et al. A short polypeptide marker sequence useful for recombinant protein identification and purification. Nat Biotechnol. 1988;6:1204.

34. Hochuli E, Bannwarth W, Dobeli H, Gentz R, Stuber D. Genetic approach to facilitate purification of recombinant proteins with a novel metal chelate adsorbant. Nat Biotechnol. 1988;6:1321.

35. Smith DB, Johnson KS. Single-step purification of polypeptides expressed in Escherichia coli as fusions with glutathione S-transferase. Gene. 1988;67:31.

36. Field J, Nikawa J, Broek D, MacDonald B, Rodgers L, Wilson IA, et al. Purification of a RAS-responsive adenylyl cyclase complex from Saccharomyces cerevisiae by use of an epitope addition method. Mol Cell Biol. 1988:8:2159.

37. Grant GA. Contrasting catalytic and allosteric mechanisms for phosphoglycerate dehydrogenases. Arch Biochem Biophys. 2012;519:175.

38. Dey S, Grant GA, Sacchettini JC. Crystal structure of Mycobacterium tuberculosis D-3-phosphoglycerate dehydrogenase: extreme asymmetry in a tetramer of identical subunits. J Biol Chem. 2005;280:14892.

39. Achouri Y, Rider MH, Schaftingen EV, Robbi M. Cloning, sequencing and expression of rat liver 3-phosphoglycerate dehydrogenase. Biochemical J. 1997;323(Pt 2):365.

40. Lund K, Merrill DK, Guynn RW. Purification and subunit structure of phosphoglycerate dehydrogenase from rabbit liver. Biochemical J. 1986;238:919.

41. Ali V, Hashimoto T, Shigeta Y, Nozaki T. Molecular and biochemical characterization of D-phosphoglycerate dehydrogenase from Entamoeba histolytica: a unique enteric protozoan parasite that possesses both phosphorylated and nonphosphorylated serine metabolic pathways. European J Biochemist/FEBS. 2004;271:2670.

42. Vander Heiden MG. Targeting cancer metabolism: a therapeutic window opens. Nat Rev Drug Discov. 2011;10:671.

\section{Submit your next manuscript to BioMed Central and take full advantage of:}

- Convenient online submission

- Thorough peer review

- No space constraints or color figure charges

- Immediate publication on acceptance

- Inclusion in PubMed, CAS, Scopus and Google Scholar

- Research which is freely available for redistribution 